\title{
Cancer testis antigens and NY-BR-1 expression in primary breast cancer: prognostic and therapeutic implications
}

\author{
Dimitrios Balafoutas ${ }^{1}$, Axel zur Hausen ${ }^{2}$, Sebastian Mayer ${ }^{1}$, Marc Hirschfeld ${ }^{1,3,4}$, Markus Jaeger ${ }^{1}$, \\ Dominik Denschlag ${ }^{1}$, Gerald Gitsch ${ }^{1}$, Achim Jungbluth ${ }^{5}$ and Elmar Stickeler ${ }^{1 *}$
}

\begin{abstract}
Background: Cancer-testis antigens (CTA) comprise a family of proteins, which are physiologically expressed in adult human tissues solely in testicular germ cells and occasionally placenta. However, CTA expression has been reported in various malignancies. CTAs have been identified by their ability to elicit autologous cellular and or serological immune responses, and are considered potential targets for cancer immunotherapy. The breast differentiation antigen NY-BR-1, expressed specifically in normal and malignant breast tissue, has also immunogenic properties. Here we evaluated the expression patterns of CTAs and NY-BR-1 in breast cancer in correlation to clinico-pathological parameters in order to determine their possible impact as prognostic factors.
\end{abstract}

Methods: The reactivity pattern of various mAbs (6C1, MA454, M3H67, 57B, E978, GAGE \#26 and NY-BR-1 \#5) were assessed by immunohistochemistry in a tissue micro array series of 210 randomly selected primary invasive breast cancers in order to study the diversity of different CTAs (e.g. MAGE-A, NY-ESO-1, GAGE) and NY-BR-1. These expression data were correlated to clinico-pathological parameters and outcome data including disease-free and overall survival.

Results: Expression of at least one CTA was detectable in the cytoplasm of tumor cells in $37.2 \%$ of the cases. NY-BR-1 expression was found in $46.6 \%$ of tumors, respectively. Overall, CTA expression seemed to be linked to adverse prognosis and $\mathrm{M} 3 \mathrm{H} 67$ immunoreactivity specifically was significantly correlated to shorter overall and disease-free survival ( $p=0.000$ and 0.024 , respectively).

Conclusions: Our findings suggest that M3H67 immunoreactivity could serve as potential prognostic marker in primary breast cancer patients. The exclusive expression of CTAs in tumor tissues as well as the frequent expression of NY-BR-1 could define new targets for specific breast cancer therapies.

Keywords: Breast Cancer, Cancer-testis Antigen, NY-BR-1, Immunotherapy, Prognosis

\section{Background}

Breast cancer is the second most common human malignancy [1]. In recent years the progress in systemic treatment modalities, especially endocrinological, immunoand chemotherapeutical strategies, have substantially reduced the proportion of women who develop metastatic disease. In the context of these advances the importance to identify prognostic and predictive markers is steadily

\footnotetext{
* Correspondence: elmar.stickeler@uniklinik-freiburg.de

${ }^{1}$ Department of Obstetrics and Gynecology, University Hospital Freiburg, Hugstetterstraße 55, Freiburg 79106, Germany

Full list of author information is available at the end of the article
}

increasing in order to avoid unnecessary adjuvant therapy regimens [2].

Cancer testis antigens (CTAs) comprise an expanding family of proteins which are normally expressed in human testicular germ cells or placental trophoblast, but not in any other normal tissue. However, CTAs are present in various malignancies [3]. More than 100 CTA-related genes and/or gene families have been identified, however their biological function remains poorly understood. CTA encoding genes which are located on chromosome $\mathrm{X}$ are referred to as CT-X antigens. Expression of these antigens has been found in diverse 
malignant human tumors including breast cancer [4]. Because of their restricted expression, CTAs are considered relevant to cancer biology and their prognostic relevance has been assessed in the recent years by several studies for various malignancies $[5,6]$. Yet the prognostic significance of CTAs in breast cancer still remains unclear.

Interestingly the presence of some CTAs such as, MAGE-A family members, GAGE and NY-ESO-1 appears to correlate with clinico-pathological parameters and prognosis in tumors, such as melanoma, non-smallcell lung cancer, multiple myeloma and other tumors [7]. CTAs are frequently recognized by cytotoxic Tlymphocytes of cancer patients or they can elicit a serological immune response in the autologous host [8]. Consequently, CTAs are regarded potential candidates for the development of anti-cancer vaccines $[9,10]$. Specifically NY-ESO-1 is able to elicit combined humoral and cell mediated immune response and considered to be the most immunogenic of the above antigens. Therefore NY-ESO-1 based vaccines have been employed in several clinical vaccination trials [11].

NY-BR-1 is a differentiation antigen of the mammary tissue, since it has been detected solely in the epithelial cells of mammary ducts and lobules, whereas NY-BR-1 expression has not been found in any other tissue [12]. Thus, NY-BR-1 appears to be a breast-specific protein.

At present only few reports on CTA expression patterns and their prognostic role in breast cancer are available with limited number of patients and clinical correlations and in part controversial findings [4,13-18]. The objective of this study was to examine the expression pattern of the aforementioned CT-antigens as well as NY-BR-1 in breast cancer and to correlate them with clinico-pathological parameters including patient outcome data. This study is the first to analyze simultaneously the expression of the CTAs and NY-BR-1 in a patient collective with long-term follow up data.

\section{Methods}

\section{Patients}

For this study 210 consecutive patients diagnosed with invasive breast cancer were enrolled, according to the ethics committee of the University Hospital Freiburg, Germany (EK-Freiburg 324/09). Standard archival paraffin blocks of primary breast cancer were retrieved from the archives of the Department of Pathology of the University Hospital Freiburg. All patients underwent surgery in the Breast Unit of the Department of Gynecology of the University Hospital Freiburg. Primary treatment consisted of radical mastectomy, modified radical mastectomy, or breast-conserving surgery including sentinel and/or axillary lymph node dissection between the years 1991 and 2001. Patients who received neoadjuvant chemotherapy, or who underwent preceding treatment at another institution or patients with a second primary tumor were excluded. Median age at the time of diagnosis was 57 years. Histopathological analyses demonstrated invasive ductal cancer in $73.8 \%$ of cases and invasive lobular subtype in $7.6 \%$. The remaining $18.6 \%$ were diagnosed as ductal/lobular, mucinous (colloid), tubular, medullary and papillary carcinomas, respectively. In 88/210 (41.9\%) patients lymph node involvement was histologically confirmed at the time of surgery. 146/210 (69.5\%) of the tumors were estrogen or progesterone receptor positive. Immunohistochemical Her2/neu overexpression was recorded in 40 (21.2\%) of the cases.

Follow up ranged from 1 to 107 months (mean 62, median 68 months), recurrences occurred in 59 (28.1\%) and deaths in 43 (20.5\%) of women, respectively. The 63 cases with technical failure in microarray mapping were excluded from the study.

\section{Materials}

Paraffin-embedded tissue blocks were used to generate tissue-microarrays (TMAs). At least three representative cores of each tumor were selected. Two specimens of normal breast as well as non neoplastic breast tissue adjacent to the lesions were used as controls. Four micron paraffin sections were stained immunohistochemically as previously described [16]. The following monoclonal antibodies (mAbs) were used: mAb 6C1 (Santa Cruz Biotechnology, Inc., Santa Cruz, USA) to several members of the MAGE-A family, mAb MA454 to MAGE-A1, mAb M3H67 also to several members of the MAGE-A family and mAb 57B to MAGE-A4 [19-21]. Next to these, the immunoreactivity of mAb E978 to NY-ESO-1 [22] and mAb \#26 (BD Biosciences Clontech, Palo Alto, USA) to GAGE was assessed. For the detection of NY-BR-1, mAb NY-BR-1\#5 previously generated by our group was utilized [23].

Evaluation of the immunohistochemical staining was performed in a blinded set up regarding the clinical data. Scoring of the expression was performed semiquantitatively as described previously [24]. In brief, both percentage of stained cells and staining intensity were evaluated. No staining or weak staining in $<5 \%$ of cells was defined as 0 , weak staining in at least $5 \%$ as 1 , moderate staining in up to $50 \%$ as 2 and moderate staining in $>50 \%$ of cells and strong staining of any percentage of the cells as 3 . The results were subsequently dichotomized for statistical analysis and the defined cut-off point for positivity for the statistical analysis was set to 2 .

Our data were analysed using the statistical package SPSS for windows version 17.0 (SPSS, Chicago, Illinois, USA). The relationship among clinico-pathological parameters and CTA expression were tested using the chi-square and Fisher's exact test. Survival outcomes 
were analysed with Kaplan-Meier survival functions and compared between groups with the log-rank statistics. To determine the association of clinico-pathological parameters with survival, univariate and multivariate Cox regression models were used. The multivariate Cox regression model was adjusted for any known prognostic variables with $\mathrm{p}<0.05$. For all tests $\mathrm{p}<0.05$ was accepted as threshold of statistical significance. Cases with missing microarrays for some of the antigens were handled in the statistical analysis as missing data.

\section{Results}

\section{Expression of CTAs and NY-BR1 in invasive breast cancer}

Overall, CTA expression was restricted to neoplastic breast tissues and detected in 54 tumor samples (37.2\%) (Table 1). The expression was mainly restricted to the cytoplasm and only occasionally located in the nuclei (Figure 1). A heterogeneous expression pattern was observed regarding the percentage of positive tumor cells.

MA454 reactivity (MAGE-A1) was found in 21 of cases (15\%). In the 14 cases with moderate staining $(10.0 \%)$ this was restricted solely to the cytoplasm, whereas in the $7(5.0 \%)$ cases with strong staining both the nuclei and the cytoplasm were positive.

E978 reactivity (NY-ESO-1) was also found in 21 of cases (15\%). In $15(10.7 \%)$ cases the staining was of moderate intensity with cytoplasmic localisation and in 6 (4.3\%) samples it was strong cytoplasmatic with occasional nuclear participation.

M3H67 reactivity was detected in 17 (12.9\%) of cases. Moderate staining was observed in $8(6.1 \%)$ and strong staining in $9(6.8 \%)$ of cases. On the cellular level, in the cases of moderate staining the localisation was predominantly cytoplasmic and in the cases of strong staining it was both cytoplasmic and nuclear.

MAb \#26 reactivity (GAGE) was detected in 17 (12.8\%) of cases analysed. GAGE localisation was primarily cytoplasmic with some rare nuclear participation. Moderate

\begin{tabular}{|c|c|c|}
\hline & $\mathrm{n}$ & $\%$ \\
\hline MAGE-A1 MA 454 & $21(140)$ & 15 \\
\hline mAb M3H67 & $17(132)$ & 12,9 \\
\hline mAb 57B & $6(133)$ & 4,5 \\
\hline NY-ESO-1 E978 & $21(140)$ & 15 \\
\hline GAGE \#26 & $17(133)$ & 12,8 \\
\hline MAGE-A 6C1 & $7(141)$ & 5 \\
\hline NY-BR-1 \#5 & $61(131)$ & 46,6 \\
\hline
\end{tabular}

$\mathrm{n}=$ number of cases with antigen positivity, in parenthesis total number with successful TMA mapping for each antigen. expression was found in $9(6.8 \%)$ and strong expression in $8(6.0 \%)$ of cases.

$57 \mathrm{~B}$ reactivity (MAGE-A4) was found in $6(4.5 \%)$ of the arrays and the staining in these cases was classified as strong. Localisation was cytoplasmic with concomitant nuclear staining in approximately $20 \%$ of the nuclei in the positive areas. In $22(10.5 \%)$ of cases we observed a very weak unspecific diffuse cytoplasmic staining which was considered negative in the analyses.

Seven $(5.0 \%)$ cases revealed $\mathrm{mAb} 6 \mathrm{C} 1$ reactivity. In 3 $(2.1 \%)$ of cases the staining was moderate, predominately cytoplasmic and to a lesser extend nuclear and in 4 $(2.8 \%)$ it was strong, with both nuclear and cytoplasmic expression.

The breast differentiation antigen NY-BR-1 was immunohistochemically detected with the \#5 Mab in the ductal and lobular cells of all included non neoplastic tissues as well as in 61 of 131 cases of cancer (46.6\%). Its expression was predominantly cytoplasmic in the normal and in the tumorous tissue with nuclear participation of varying degree. The staining intensity was classified as moderate in $39(29.8 \%)$ and as strong in 22 (16.8\%) of cases. Some areas with dot-like staining pattern in the cytoplasm were also observed. No correlation was found between expression of CTAs and NY-BR-1.

\section{Correlations with clinico-pathological parameters}

The expression data of each CTA were grouped based on clinico-pathological characteristics (Table 2): Age group (in comparison to median), tumor size and grade, lymph node involvement, histological type, estrogen and progesterone receptor and HER2/neu status were compared among positive and negative samples for each CTA. Interestingly, we observed that CTA positivity in our cohort was restricted to grade 2 and 3 tumors and all grade 1 tumor samples were negative for all investigated CTAs. For the other examined parameters there was no significant difference between CTA positive and negative groups.

The expression frequency of NY-BR-1 was equally distributed among the groups with different tumor grading. Similarly we did not find any significant differences in the expression of NY-BR-1 related with other clinicopathological parameters.

\section{Clinical outcome analysis}

Factors associated with disease-free survival (DFS) and disease specific overall survival (OS) were analysed by Univariate Cox regression (Table 3). We observed a statistically significant negative prognostic impact for larger tumor size ( $\mathrm{p}=0.002$ for both DFS and OS) and lymph node metastases $(p=0.000$ for both DFS and OS). The expression of estrogen or progesterone receptor was accompanied by longer DFS $(\mathrm{p}=0,019)$, but for OS this 


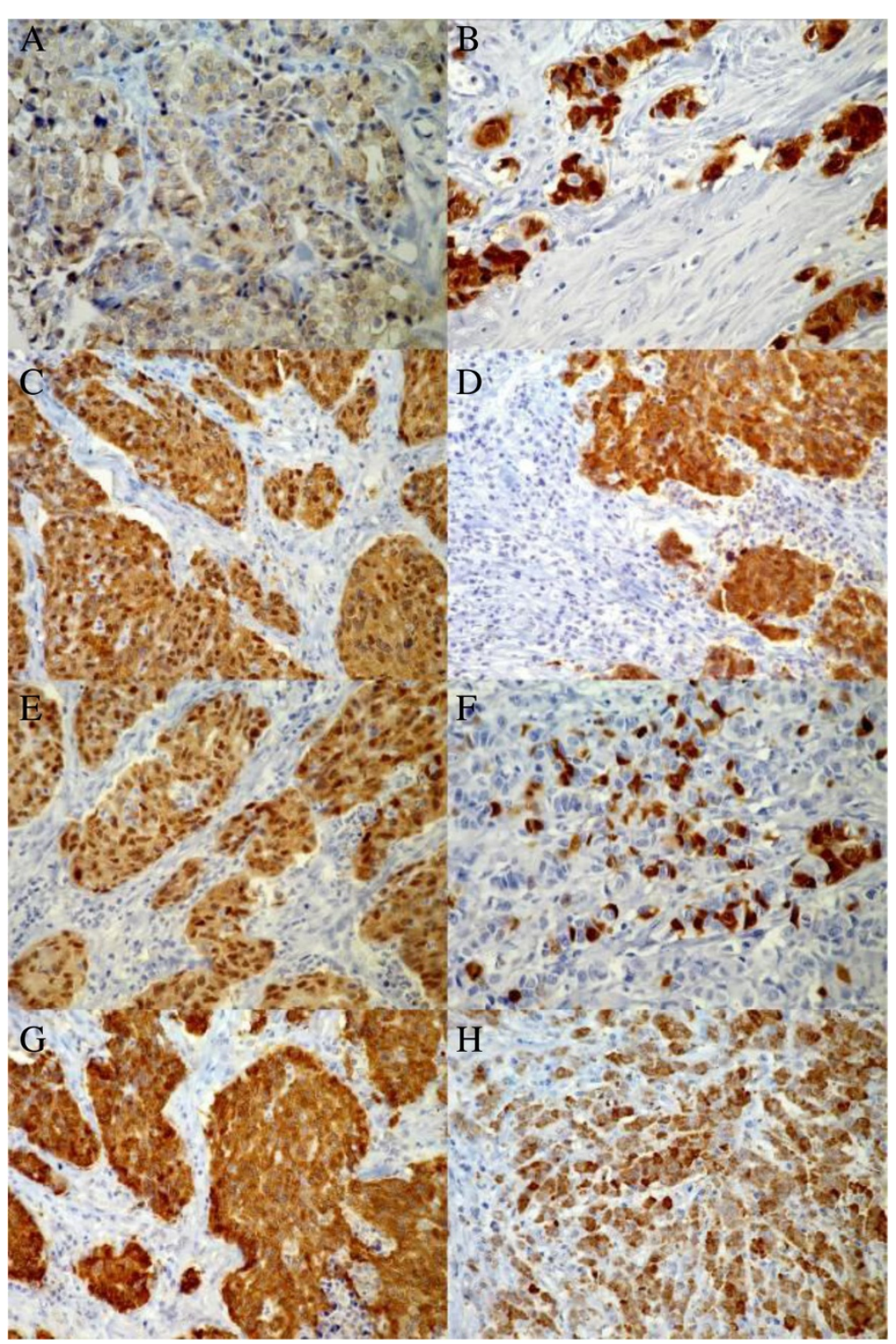

Figure 1 Immunohistochemical detection of cancer - testis antigens and NY-BR-1 in primary breast cancer tissue microarrays. A: Example of moderate staining of MAGE A1 in approximately $80 \%$ of the tumor cells. The staining is restricted to the cytoplasm. B: Strong nuclear and cytoplasmic expression of MAGE A1. C: Extensive strong nuclear and cytoplasmic M3H67 immunoreactivity. D: Strong, mainly cytoplasmic and occasionally nuclear 57B immunoreactivity. E: Extensive strong nuclear and cytoplasmic detection of MAGE A (6C1). F: Focal strong, mainly cytoplasmic staining of approximately $20 \%$ of tumor cells for GAGE. G: Strong extensive cytoplasmic and occasionally nuclear staining of NY-ESO-1. H: Strong cytoplasmic NY-BR-1 staining of approximately $80 \%$ of cells with scarce nuclear detection (40x objective).

correlation did not reach statistical significance. In the univariate Cox regression analysis NY-BR-1 did not seem to affect recurrence or survival.

In contrast to NY-BR-1, Kaplan-Meier survival analysis (Figure 2) demonstrated a strong clinical impact on survival for the immunoreactivity pattern of most of the examined CTAs. The detected adverse effects were statistically significant for both recurrence and disease related death for $\mathrm{M} 3 \mathrm{H} 67$ (p-log rank=0.004 and 0.000) and 57B (p-log rank=0.015 and 0.036) immunoreactivity, respectively. MAGE-A1 positive patients had a shorter OS (p-log rank =0.028), but no impact on DFS was observed. Additionally we found a clear, though statistically not significant trend for negative effects of mAb \#26 (GAGE) and mAb 6C1 (MAGE-A family) expression on DFS and OS: GAGE positive patients had a $19.9 \%$ shorter DFS and a $14.7 \%$ shorter OS ( $\mathrm{p}$-log rank $=0.090$ and 0.238 ) and $6 \mathrm{C} 1$ positive patients a $23.19 \%$ shorter DFS and a $16.97 \%$ shorter OS (p-log rank $=0.090$ and 0.453). 
Table 2 Clinicopathological characteristics of breast cancer patients in our collective

\begin{tabular}{lll}
\hline & & $\mathbf{n}(\%)$ \\
\hline Age & >median & $105(50 \%)$ \\
Tumor grade & 1 & $105(50 \%)$ \\
& 2 & $7(3,50 \%)$ \\
Tumor size & 3 & $92(45,80 \%)$ \\
& pT1 & $102(50,70 \%)$ \\
Lymph node status & pT2 & $88(46,60 \%)$ \\
Histological type & pT3/4 & $78(41,30 \%)$ \\
& pNo & $23(12,20 \%)$ \\
ER/PR status & pN1/2/3 & $119(57,50 \%)$ \\
& ductal & $88(42,50 \%)$ \\
HER2/neu & lobular & $75(58,10 \%)$ \\
& other & $16(12,40 \%)$ \\
& negative & $38(29,40 \%)$ \\
& positive & $58(28,40 \%)$ \\
& 0 & $146(71,60 \%)$ \\
& 1 & $54(28,60 \%)$ \\
& 2 & $84(44,40 \%)$ \\
& 3 & $11(5,80 \%)$ \\
& & $40(21,20 \%)$ \\
\hline
\end{tabular}

Multivariate analysis identifies $\mathrm{M} 3 \mathrm{H} 67$ reactivity as a strong prognosticator for overall survival

In order to identify the independent prognostic factors in our cohort we performed a multivariate Cox regression analysis (Table 4). Lymph node status was confirmed as a known independent prognostic parameter with a hazard ratio (HR) 6.37 (95\% CI 2.6-17.4, $\mathrm{p}=0.0001)$ and 5.99 (95\% CI 1.9-18.7, p=0.002) for DFS and OS respectively. However, M3H67 reactivity exhibited the strongest prognostic impact in this study, with a HR of 7.69 (95\% CI 2.6-22.8, $\mathrm{p}=0.0001$ ) for OS and the second strongest for DFS with a HR of 4.36 (95\% CI 1.2-15.6, p=0.024). Estrogen or progesterone receptor positivity was correlated with decreased risk of disease recurrence (HR 0.40, 95\% CI $0.1-0.8, \mathrm{p}=0.015)$ but was not included in the multivariate analysis for overall survival, because it did not reach the significance threshold in univariate analysis.

\section{Discussion}

The expression of CTAs has been described in several malignant tumors [5,6,25-27] CTAs have been identified in melanomas, non-small cell lung and pancreatic cancer, serous ovarian cancer, hepatocellular carcinomas, multiple myelomas as well as in breast cancer [17]. The CTA expression frequency in breast cancer varies in the literature reaching up to $88 \%$ [14]. However, the reproducibility of the studies suffers in terms of standardization regarding tumor specimen (primary tumors or metastases), methodology (RT-PCR, Western-blot or immunohistochemistry), and the evaluation of the IHC-staining.

Using a broad spectrum of diverse mAbs, we found a total percentage for the presence of any CTA of 37.6\%, which is in accordance with most of the existing reports [14]. However, our cohort included solely tissues from primary tumors and in contrast to other reports we valued all cases with weak staining as negative. One study [4] found a positivity of $47 \%$ in primary breast

Table 3 Univariate-Cox-regression-analysis of known prognostic factors CTAs and NY-BR-1 of breast-cancer patients

\begin{tabular}{|c|c|c|c|c|c|c|}
\hline & & DFS & & & OS & \\
\hline & HR & $95 \% \mathrm{Cl}$ & $\mathbf{p}$ & HR & $95 \% \mathrm{Cl}$ & $\mathbf{P}$ \\
\hline Age vs median & 0,884 & $0,530-1,475$ & 0,637 & 0,987 & $0,542-1,796$ & 0,966 \\
\hline Tumor grade & 1,521 & $0,930-2,488$ & 0,095 & 1,652 & $0,903-3,022$ & 0,104 \\
\hline Tumor stage & 1,808 & $1,241-2,632$ & 0,002 & 1,988 & $1,292-3,058$ & 0,002 \\
\hline Lymph node status & 2,971 & $1,730-5,104$ & 0 & 3,348 & $1,714-6,537$ & 0 \\
\hline Histological type & 0,864 & $0,672-1,111$ & 0,255 & 0,855 & $0,624-1,170$ & 0,327 \\
\hline ER/PR status & 0,526 & $0,307-0,901$ & 0,019 & 0,775 & $0,399-1,505$ & 0,451 \\
\hline HER2/neu status & 1,064 & $0,826-1,369$ & 0,632 & 0,986 & $0,727-1,337$ & 0,929 \\
\hline MAGE A1 MA454 & 1,278 & $0,564-2,898$ & 0,557 & 2,284 & $0,910-5,732$ & 0,078 \\
\hline M3H67 reactivity & 2,85 & $1,350-6,017$ & 0,006 & 4,27 & $1,834-9,941$ & 0,001 \\
\hline 57B reactivity & 3,406 & $1,15-10,03$ & 0,026 & 3,446 & $1,00-11,77$ & 0,049 \\
\hline NY-ESO-1 E978 & 1,272 & $0,563-2,877$ & 0,563 & 0,805 & $0,242-2,684$ & 0,724 \\
\hline GAGE \#26 & 1,988 & $0,875-4,516$ & 0,101 & 1,98 & $0,739-5,303$ & 0,174 \\
\hline MAGE A 6C1 & 1,65 & $0,498-5,466$ & 0,413 & 1,876 & $0,435-8,087$ & 0,399 \\
\hline NY-BR-1 \#5 & 1,522 & $0,816-2,839$ & 0,186 & 1,235 & $0,554-2,752$ & 0,606 \\
\hline
\end{tabular}

Abbreviations: DFS Disease free survival; OS Overall survival; HR Hazard ratio; Cl Confidence interval. 

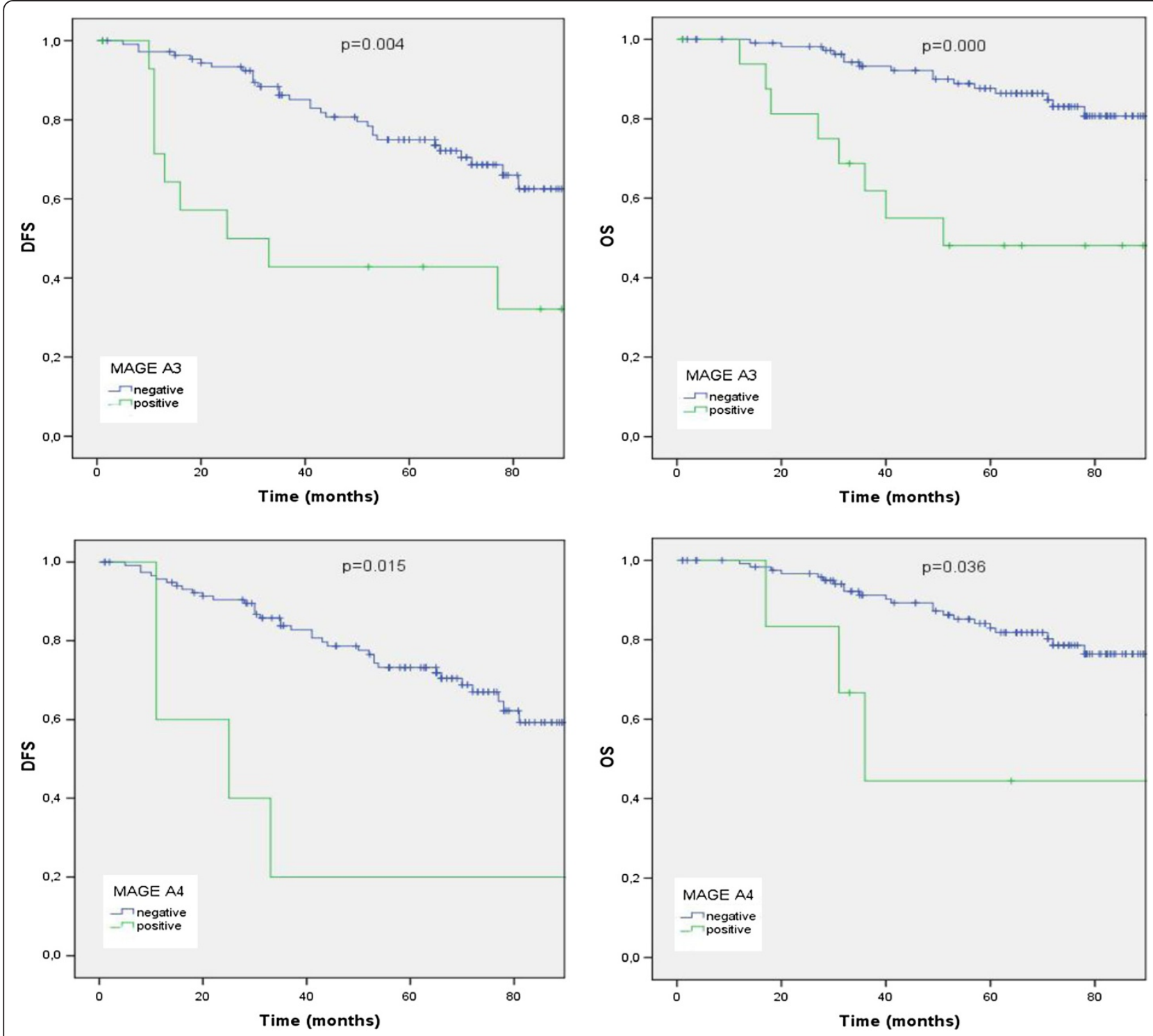

Figure 2 Kaplan Meier survival analysis for disease-free (DFS) and overall-survival (OS): In the presence (green line) or absence (blue line) of immunohistochemical reactivity of M3H67 and 57B. p: log rank test.

tumors, however, including also the tumors with $1-2 \%$ positive stained cells. The same authors reported a significant higher percentage of CTA expression in metastatic tumors (66\%). These findings fit very well into the tumorbiological context of this gene familiy and reflect their potential role as tumor associated antigens in tumor progression. The antibodies tested in our study revealed the same distribution pattern, concerning isolated cells or groups of cells, differing, however, in the degree of expression. MAGE-A1 and NY-ESO-1 were detected at higher frequency and we recorded neither a significant coexpression nor a mutual exclusion of the various CTAs, in accordance with the literature. However, we could not confirm the reported higher expression of CTAs in estrogen receptor negative cases. Our findings of a clear restriction of CTA expression to grade 2 and 3 cancers is in concordance with other studies [28], however, the small number of grade 1 tumors did not allow us to perform a reliable statistical analysis in this case.

CTA expression was recently associated to prognosis with an adverse impact in gastrointestinal stromal tumors [25], oral squamous cell carcinomas [29], multiple myelomas [30], and cervical cancers [31]. However, controversial findings were also reported correlating CTAs with a less aggressive tumor behaviour [32,33]. Our findings demonstrate a clear association for CTA expression and prognosis. Of all the antibodies tested in our study, M3H67 reactivity seems to exhibit the strongest prognostic impact for the course of breast cancer. 
Table 4 Multivariate-Cox-regression-analysis for disease-free survival and overall-survival of breast-cancer patients

\begin{tabular}{|c|c|c|c|c|c|c|}
\hline & \multicolumn{3}{|c|}{ DFS } & \multicolumn{3}{|c|}{ OS } \\
\hline & HR & $95 \% \mathrm{Cl}$ & $p$ & HR & $95 \% \mathrm{Cl}$ & $\mathbf{P}$ \\
\hline Tumor stage & 0,807 & $0,435-1,496$ & 0,496 & 1,393 & $0,658-2,948$ & 0,386 \\
\hline Lymph node status & 6,737 & $2,607-17,409$ & 0,000 & 5,99 & $1,920-18,688$ & 0,002 \\
\hline ER/PR status & 0,405 & $0,196-0,837$ & 0,015 & & & \\
\hline M3H67 reactivity & 4,355 & $1,218-15,572$ & 0,024 & 7,693 & $2,597-22,786$ & 0,000 \\
\hline 57B reactivity & 1,328 & $0,229-7,713$ & 0,752 & 0,71 & $0,120-4,216$ & 0,706 \\
\hline
\end{tabular}

Abbreviations: DFS Disease free survival; OS Overall survival; HR Hazard ratio; Cl Confidence interval.

MAGE-A proteins bind to KAP1 which is a repressor of p53 and suppress apoptosis in MAGE-A expressing cell lines [34]. Small interfering RNA (siRNA) suppression of MAGE genes leads to increased p53 expression and increased apoptosis in melanoma cell lines [34], thus the overexpression of MAGE proteins in breast cancer could also protect malignant cells from programmed cell death. For MAGE-A3, specifically, a reverse correlation is shown in pituitary tumors between tumor supressive FGFR2 and MAGE-A3 mRNA expression [35], where siRNA down-regulation of MAGE-A3 results in p53 promoter activation and reduced cell proliferation. GAGE proteins seem to have a similar function, since its transfection can render cells resistant against interferongamma or death receptor Fas/CD95/APO-1 induced apoptosis [36]. Clinically, overexpression of these proteins seems, indeed, to correlate with adverse prognosis. Due to the fact, that CTAs are relatively widely expressed, this marker could give the additional information for a substantial proportion of breast cancer patients. $57 \mathrm{~B}$ reactivity had a prognostic relevance in univariate analysis, however, it could not be validated as an independent prognostic factor in the multivariate approach. This limitation might be due to the relatively small number of cases available for statistical analyses. 57B immunoreactivity has been previously associated with poor prognosis in cholangiocarcinoma [37]. Additionally, M3H67 immunoreactivity, as a marker for MAGE-A expression, mainly MAGE-A3, was found to be associated with poor prognosis in gastrointestinal stromal tumors [38]. Moreover MAGE-A3 expression detected with RT-PCR had an adverse prognostic effect in non-small-cell lung cancers [39]. Most previous studies also recognized an adverse correlation of MAGE A family antigens either to the survival or indirectly to established prognostic factors [4,40], with a unique report of MAGE-A4 to be a favourable prognostic factor [33].

In the development of vaccines against breast cancer two major target antigen groups have been proposed: CTAs because of their unique expression pattern in tumor, but not in normal tissue and the breast differentiation antigens. Although our lack of knowledge about the biological function of CTAs complicates their utilisation, the use of CTAs as targets for the vaccination of breast cancer has been under debate widely the last years [41].

The exact biological function of NY-ESO-1 remains unknown. However recent experiments indicate a possible relevance of NY-ESO-1 expression for DNA-methylation. [42]. The frequent expression of NY-ESO-1 in our cohort could play a potential role in the application of additional immunological therapies in breast cancer, since it has been demonstrated that NY-ESO-1 can elicit strong CD8 and CD4 T-cell response in seropositive patients $[15,43,44]$. Therefore it has been target of several vaccination efforts in the past [11]. In vivo the T-cell responses against tumor-associated antigens seem to improve the prognosis in hepatocellular carcinoma [45]. However, suppression of the immune response via regulatory T-cells has also been described [46]. Several clinical trials [47] have been performed on vaccines targeting breast cancer and two new trials are now recruiting for the use of CTAs as targets. A recent study [16] has showed that CTA expression is more frequent in triple negative breast cancer. This is of particular interest, since our conventional adjuvant therapeutic possibilities in this subgroup of breast cancer are limited.

An important consideration when conducting immunohistochemical studies on the MAGE-A family proteins is their high homology. Cross-reactivity of antibodies to MAGE-A CTAs cannot be ruled out. At this point solely mAb MA454 to MAGE-A1 can be regarded as truly specific for a particular MAGE-A antigen. Attempts to generate reagents to other MAGE-A family members such as MAGE-A3, the most prevalent MAGE-A antigen on a molecular level, have rendered mixed results. This is best exemplified by mAb $57 \mathrm{~B}$, which was originally generated as a MAGE-A3 reagent [20]. Subsequent analysis indicated reactivity with several MAGE-A family members [19]. More recent data indicate reactivity of $\mathrm{mAb} 57 \mathrm{~B}$ to MAGE-A4 [48]. The same applies to mAb M3H67 which was originally generated to MAGE-A3 but is now considered reactive with several members of the MAGE-A family (unpublished data). However this does not necessarily negatively impact the prognostic value of immunohistochemistry, but it complicates the identification of the best target for cancer immunotherapy. 
Also we expected that positivity for mAb $6 \mathrm{C} 1$, which reacts with several MAGE-A antigens, would be more frequent and comparable to the other anti-MAGE-A reagents. However, in our series this was not the case. This could be based on different affinities of the various reagents for similar antigens generating incongruent staining patters in spite of overlapping specifity patterns.

NY-BR-1 can be identified at the protein level in physiological as well as cancerous breast tissue [49] although recently it has been also described in a vulvar lesion [50]. The function of NY-BR-1 in vivo has not yet been clarified. Bioinformatics analyses showing a DNAbinding site followed by a leucine zipper motif suggest that this molecule acts as a transcription factor. Because of five tandem ankyrin repeats NY-BR-1 could also have a role in protein-protein interactions [49]. Our data suggest that NY-BR-1 is strongly expressed in a great proportion of primary breast cancers (46.6\%). This frequent expression of NY-BR-1 has been previously described [12]. Humoral immune response against endogenous NY-BR-1 has been confirmed by detecting the spontaneous NY-BR-1 directed antibody responses in breast cancer patients, tested positive for NY-BR-1 by RT-PCR [51]. Additionally two HLA-A2 restricted peptide epitopes for NY-BR-1 that were recognized by CD8 $+\mathrm{T}$ cells derived from breast cancer patients have been defined [52]. Due to the restricted expression pattern, combined with the wide expression in tumors, NY-BR-1 seems to be an ideal potential target for innovative immunotherapeutic approaches of breast cancer because of the more frequent expression in comparison to HER2/neu, the current reference target for cancer immunotherapy. This approach exerts even more potential since we could not confirm a recently reported correlation between NY-BR-1 and HER2/ neu expression [53].

Our analyses did not show any significant coexpression of NY-BR-1 with the CT-antigens, neither a mutual exclusion. Since M3H67 reactivity was associated with tumor progression while NY-BR-1 represents a differentiation antigen it might be possible that these tumors with a high $\mathrm{M} 3 \mathrm{H} 67$ reactivity and simultaneous absence of NY-BR-1 expression behave in a tumorbiological aggressive fashion. In our cohort, we observed six such cases with an indeed high mortality rate (50\%), however the number of cases was too small to extract any further conclusions.

In total $60.3 \%$ of our patients were positive for either CT-antigens or NY-BR-1 or both. Theoretically this could facilitate polyvalent vaccines containing more than one antigen in order to achieve in parallel targeting of a higher percentage of tumor cells in genetically heterogeneous tumors, or vaccines that can be used without prior antigen monitoring. The highly immunogenic potential of CT-antigens combined with immune response adjuvants [11] is not yet fully explored but appears promising.

\section{Conclusions}

To our knowledge this study is the largest retrospective analysis of the expression and prognostic role of numerous CT-antigens and NY-BR-1 in breast cancer. Despite the above limitations we believe that our results underline the emerging role of the above group of genes for prognosis and therapeutical approaches in breast cancer in the future. Especially mAb M3H67 reactivity, probably reflecting presence of several MAGE-A antigens was proven as a strong independent prognostic factor. The relatively small number of patients may have concealed other important clinical correlations that appeared only as trends. Therefore a prospective study with a much greater number of patients and the possibility of stratification according to primary and adjuvant therapy is imperatively needed.

\section{Competing interests}

All authors declare to have no financial or non-financial competing interests. There is no funding source to be disclosed.

\section{Authors' contributions}

DB participated in the array analysis, performed with DD the statistical analysis and drafted the manuscript. ES and AzH conceived the study and participated in its design and coordination. AzH additionally performed the pathological evaluation of the specimens and participated in the array analysis. SM was responsible for the recruitment of the patients in the study and obtained the informed consent. MJ and SM generated the tissue microarrays. AJ provided the monoclonal antibodies and carried out the immunochistochemical staining. $\mathrm{MH}$ contributed to the evaluation of the results. GG coordinated the team and made the final corrections. All authors read and approved the final manuscript.

\section{Author details}

'Department of Obstetrics and Gynecology, University Hospital Freiburg, Hugstetterstraße 55, Freiburg 79106, Germany. '2Department of Pathology, GROW- School for Oncology and Developmental Biology, Maastricht University Medical Center, Postbus 5800, Maastricht 6202 AZ, The Netherlands. ${ }^{3}$ German Cancer Consortium (DKTK), Heidelberg, Germany. ${ }^{4}$ German Cancer Research Center (DKFZ), Heidelberg, Germany. ${ }^{5}$ Ludwig Institute for Cancer Research, New York Branch at Memorial Sloan-Kettering Cancer Center, 1275 York Avenue, BOX 32, New York, NY 10021-6007, USA.

Received: 4 March 2013 Accepted: 22 May 2013

Published: 3 June 2013

\section{References}

1. Centers for Disease Control and Prevention: Breast Cancer Statistics: http://www.cdc.gov/cancer/breast/statistics/.

2. Stickeler E: Prognostic and predictive markers for treatment decisions in early breast cancer. Breast Care 2011, 6:193-198.

3. van der Bruggen P, Traversari C, Chomez P, Lurquin C, De Plaen E, Van den Eynde $B$, Knuth $A$, Boon $T$ : A gene encoding an antigen recognized by cytolytic T lymphocytes on a human melanoma. Science 1991, 254:1643-1647.

4. Grigoriadis A, Caballero OL, Hoek KS, da Silva L, Chen YT, Shin SJ, Jungbluth AA, Miller LD, Clouston D, Cebon J, et al: CT-X antigen expression in human breast cancer. Proc Natl Acad Sci USA 2009, 106:13493-13498.

5. Atanackovic D, Luetkens T, Hildebrandt Y, Arfsten J, Bartels K, Horn C, Stahl T, Cao Y, Zander AR, Bokemeyer C, Kroger N: Longitudinal analysis and prognostic effect of cancer-testis antigen expression in multiple myeloma. Clin Cancer Res 2009, 15:1343-1352. 
6. Yoshida N, Abe H, Ohkuri T, Wakita D, Sato M, Noguchi D, Miyamoto M, Morikawa T, Kondo S, Ikeda H, Nishimura T: Expression of the MAGE-A4 and NY-ESO-1 cancer-testis antigens and T cell infiltration in non-small cell lung carcinoma and their prognostic significance. Int J Oncol 2006, 28:1089-1098

7. Caballero OL, Chen YT: Cancer/testis (CT) antigens: potential targets for immunotherapy. Cancer Sci 2009, 100:2014-2021.

8. Stockert E, Jager E, Chen YT, Scanlan MJ, Gout I, Karbach J, Arand M, Knuth A, Old LJ: A survey of the humoral immune response of cancer patients to a panel of human tumor antigens. J Exp Med 1998, 187:1349-1354.

9. Karbach J, Gnjatic S, Bender A, Neumann A, Weidmann E, Yuan J, Ferrara CA, Hoffmann E, Old LJ, Altorki NK, Jager E: Tumor-reactive CD8+ T-cell responses after vaccination with NY-ESO-1 peptide, CpG 7909 and montanide ISA-51: association with survival. Int J Cancer 2010, 126:909-918.

10. Bioley G, Dousset C, Yeh A, Dupont B, Bhardwaj N, Mears G, Old LJ, Ayyoub $M$, Valmori D: Vaccination with recombinant NY-ESO-1 protein elicits immunodominant HLA-DR52b-restricted CD4+ T cell responses with a conserved T cell receptor repertoire. Clin Cancer Res 2009, 15:4467-4474.

11. Valmori D, Souleimanian NE, Tosello V, Bhardwaj N, Adams S, O'Neill D, Pavlick A, Escalon JB, Cruz CM, Angiulli A, et al: Vaccination with NY-ESO-1 protein and $\mathrm{CpG}$ in montanide induces integrated antibody/Th1 responses and CD8 T cells through cross-priming. Proc Natl Acad Sci USA 2007, 104:8947-8952.

12. Jager D, Filonenko V, Gout I, Frosina D, Eastlake-Wade S, Castelli S, Varga Z, Moch $\mathrm{H}$, Chen YT, Busam KJ, et al: NY-BR-1 is a differentiation antigen of the mammary gland. Appl Immunohistochem Mol Morphol 2007, 15:77-83.

13. Theurillat JP, Ingold F, Frei C, Zippelius A, Varga Z, Seifert B, Chen YT, Jager $D$, Knuth A, Moch H: NY-ESO-1 protein expression in primary breast carcinoma and metastases: correlation with CD8+ T-cell and CD79a+ plasmacytic/B-cell infiltration. Int J Cancer 2007, 120:2411-2417.

14. Mischo A, Kubuschok B, Ertan K, Preuss KD, Romeike B, Regitz E, Schormann C, de Bruijn D, Wadle A, Neumann F, et al: Prospective study on the expression of cancer testis genes and antibody responses in 100 consecutive patients with primary breast cancer. Int J Cancer 2006, 118:696-703.

15. Sugita $Y$, Wada H, Fujita S, Nakata T, Sato S, Noguchi $Y$, Jungbluth AA, Yamaguchi M, Chen YT, Stockert E, et al: NY-ESO-1 expression and immunogenicity in malignant and benign breast tumors. Cancer Res 2004, 64:2199-2204.

16. Curigliano G, Viale G, Ghioni M, Jungbluth AA, Bagnardi V, Spagnoli GC, Neville AM, Nole F, Rotmensz N, Goldhirsch A: Cancer-testis antigen expression in triple-negative breast cancer. Ann Oncol 2011, 22:98-103.

17. Kruger $\mathrm{S}$, Ola $\mathrm{V}$, Feller AC, Fischer D, Friedrich M: Expression of cancertestis antigen CT7 (MAGE-C1) in breast cancer: an immunohistochemical study with emphasis on prognostic utility. Pathol Oncol Res 2007, 13:91-96.

18. Jager D, Stockert E, Scanlan MJ, Gure AO, Jager E, Knuth A, Old L, Chen YT: Cancer-testis antigens and ING1 tumor suppressor gene product are breast cancer antigens: characterization of tissue-specific ING1 transcripts and a homologue gene. Cancer Res 1999, 59:6197-6204.

19. Rimoldi D, Salvi S, Schultz-Thater E, Spagnoli GC, Cerottini JC: Anti-MAGE-3 antibody 57B and anti-MAGE-1 antibody $6 \mathrm{C} 1$ can be used to study different proteins of the MAGE-a family. Int J Cancer 2000, 86:749-751.

20. Kocher T, Schultz-Thater E, Gudat F, Schaefer C, Casorati G, Juretic A, Willimann T, Harder F, Heberer M, Spagnoli GC: Identification and intracellular location of MAGE-3 gene product. Cancer Res 1995, 55:2236-2239

21. Jungbluth AA, Stockert E, Chen $Y T$, Kolb D, Iversen $K$, Coplan $K$, Williamson B, Altorki N, Busam KJ, Old LJ: Monoclonal antibody MA454 reveals a heterogeneous expression pattern of MAGE-1 antigen in formalin-fixed paraffin embedded lung tumours. Br J Cancer 2000, 83:493-497.

22. Jungbluth AA, Chen YT, Stockert E, Busam KJ, Kolb D, Iversen K, Coplan K, Williamson B, Altorki N, Old LJ: Immunohistochemical analysis of NY-ESO-1 antigen expression in normal and malignant human tissues. Int $J$ Cancer 2001, 92:856-860.

23. Varga Z, Theurillat JP, Filonenko V, Sasse B, Odermatt B, Jungbluth AA, Chen $Y T$, Old $L$, Knuth A, Jager D, Moch $H$ : Preferential nuclear and cytoplasmic NY-BR-1 protein expression in primary breast cancer and lymph node metastases. Clin Cancer Res 2006, 12:2745-2751.

24. van Diest PJ, van Dam P, Henzen-Logmans SC, Berns E, van der Burg ME, Green J, Vergote I: A scoring system for immunohistochemical staining: consensus report of the task force for basic research of the EORTC-GCCG . European organization for research and treatment of cancergynaecological cancer cooperative group. J Clin Pathol 1997, 50:801-804

25. Perez D, Herrmann T, Jungbluth AA, Samartzis P, Spagnoli G, Demartines N, Clavien PA, Marino S, Seifert B, Jaeger D: Cancer testis antigen expression in gastrointestinal stromal tumors: new markers for early recurrence. Int J Cancer 2008, 123:1551-1555.

26. Velazquez EF, Jungbluth AA, Yancovitz M, Gnjatic S, Adams S, O'Neill D, Zavilevich K, Albukh T, Christos P, Mazumdar M, et al: Expression of the cancer/testis antigen NY-ESO-1 in primary and metastatic malignant melanoma (MM)correlation with prognostic factors. Cancer Immun 2007, 7:11.

27. Tammela J, Jungbluth AA, Qian F, Santiago D, Scanlan MJ, Keitz B, Driscoll D, Rodabaugh K, Lele S, Old LJ, Odunsi K: SCP-1 cancer/testis antigen is a prognostic indicator and a candidate target for immunotherapy in epithelial ovarian cancer. Cancer Immun 2004, 4:10.

28. Chen YT, Ross DS, Chiu R, Zhou XK, Chen YY, Lee P, Hoda SA, Simpson AJ, Old LJ, Caballero O, Neville AM: Multiple cancer/testis antigens are preferentially expressed in hormone-receptor negative and high-grade breast cancers. PLoS One 2011, 6:e17876.

29. Ries J, Mollaoglu N, Vairaktaris E, Neukam FW, Nkenke E: Diagnostic and therapeutic relevance of NY-ESO-1 expression in oral squamous cell carcinoma. Anticancer Res 2009, 29:5125-5130.

30. Andrade VC, Vettore AL, Felix RS, Almeida MS, Carvalho F, Oliveira JS Chauffaille ML, Andriolo A, Caballero OL, Zago MA, Colleoni GW: Prognostic impact of cancer/testis antigen expression in advanced stage multiple myeloma patients. Cancer Immun 2008, 8:2

31. Napoletano C, Bellati F, Tarquini E, Tomao F, Taurino F, Spagnoli G, Rughetti A, Muzii L, Nuti M, Benedetti Panici P: MAGE-a and NY-ESO-1 expression in cervical cancer: prognostic factors and effects of chemotherapy. Am J Obstet Gynecol 2008, 198:e91-e99.

32. Grau E, Oltra S, Martinez F, Orellana C, Canete A, Fernandez JM, HernandezMarti M, Castel V: MAGE-A1 expression is associated with good prognosis in neuroblastoma tumors. J Cancer Res Clin Oncol 2009, 135:523-531.

33. Bandic D, Juretic A, Sarcevic B, Separovic V, Kujundzic-Tiljak M, Hudolin T, Spagnoli GC, Covic D, Samija M: Expression and possible prognostic role of MAGE-A4, NY-ESO-1, and HER-2 antigens in women with relapsing invasive ductal breast cancer: retrospective immunohistochemical study. Croat Med J 2006, 47:32-41.

34. Yang B, O'Herrin SM, Wu J, Reagan-Shaw S, Ma Y, Bhat KM, Gravekamp C, Setaluri $V$, Peters N, Hoffmann FM, et al: MAGE-a, mMage-b, and MAGE-C proteins form complexes with KAP1 and suppress p53-dependent apoptosis in MAGE-positive cell lines. Cancer Res 2007, 67:9954-9962.

35. Zhu X, Asa SL, Ezzat S: Fibroblast growth factor 2 and estrogen control the balance of histone 3 modifications targeting MAGE-A3 in pituitary neoplasia. Clin Cancer Res 2008, 14:1984-1996.

36. Cilensek ZM, Yehiely F, Kular RK, Deiss LP: A member of the GAGE family of tumor antigens is an anti-apoptotic gene that confers resistance to Fas/CD95/APO-1, interferon-gamma, taxol and gamma-irradiation. Cancer Biol Ther 2002, 1:380-387.

37. Zhou JX, Li Y, Chen SX, Deng AM: Expression and prognostic significance of cancer-testis antigens (CTA) in intrahepatic cholagiocarcinoma. J Exp Clin Cancer Res 2011, 30:2.

38. Perez D, Hauswirth F, Jager D, Metzger U, Samartzis EP, Went $P$, Jungbluth A: Protein expression of cancer testis antigens predicts tumor recurrence and treatment response to imatinib in gastrointestinal stromal tumors. Int J Cancer 2011, 15:2947-2952.

39. Sienel W, Mecklenburg I, Dango S, Ehrhardt P, Kirschbaum A, Passlick B, Pantel K: Detection of MAGE-a transcripts in bone marrow is an independent prognostic factor in operable non-small-cell lung cancer. Clin Cancer Res 2007, 13:3840-3847.

40. Otte M, Zafrakas M, Riethdorf L, Pichlmeier U, Loning T, Janicke F, Pantel K: MAGE-a gene expression pattern in primary breast cancer. Cancer Res 2001, 61:6682-6687.

41. Taylor M, Bolton LM, Johnson P, Elliott T, Murray N: Breast cancer is a promising target for vaccination using cancer-testis antigens known to elicit immune responses. Breast Cancer Res 2007, 9:R46.

42. Rao M, Chinnasamy N, Hong JA, Zhang Y, Zhang M, Xi S, Liu F, Marquez VE, Morgan RA, Schrump DS: Inhibition of histone lysine methylation enhances cancer-testis antigen expression in lung cancer cells: implications for adoptive immunotherapy of cancer. Cancer Res 2011, 71:4192-4204. 
43. Jager E, Nagata Y, Gnjatic S, Wada H, Stockert E, Karbach J, Dunbar PR, Lee SY, Jungbluth A, Jager D, et al: Monitoring CD8 T cell responses to NYESO-1: correlation of humoral and cellular immune responses. Proc Natl Acad Sci USA 2000, 97:4760-4765.

44. Wang Y, Wu XJ, Zhao AL, Yuan YH, Chen YT, Jungbluth AA, Gnjatic S, Santiago D, Ritter G, Chen WF, et al: Cancer/testis antigen expression and autologous humoral immunity to NY-ESO-1 in gastric cancer. Cancer Immun 2004, 4:11.

45. Hiroishi K, Eguchi J, Baba T, Shimazaki T, Ishii S, Hiraide A, Sakaki M, Doi H, Uozumi S, Omori R, et al: Strong CD8(+) T-cell responses against tumorassociated antigens prolong the recurrence-free interval after tumor treatment in patients with hepatocellular carcinoma. J Gastroenterol 2010, 45:451-458

46. Gnjatic S, Altorki NK, Tang DN, Tu SM, Kundra V, Ritter G, Old LI, Logothetis CJ, Sharma P: NY-ESO-1 DNA vaccine induces T-cell responses that are suppressed by regulatory T cells. Clin Cancer Res 2009, 15:2130-2139.

47. Ko BK, Kawano K, Murray JL, Disis ML, Efferson CL, Kuerer HM, Peoples GE, loannides CG: Clinical studies of vaccines targeting breast cancer. Clin Cancer Res 2003, 9:3222-3234.

48. Landry C, Brasseur F, Spagnoli GC, Marbaix E, Boon T, Coulie P, Godelaine D: Monoclonal antibody $57 \mathrm{~B}$ stains tumor tissues that express gene MAGEA4. Int J Cancer 2000, 86:835-841.

49. Jager D, Stockert E, Gure AO, Scanlan MJ, Karbach J, Jager E, Knuth A, Old L, Chen YT: Identification of a tissue-specific putative transcription factor in breast tissue by serological screening of a breast cancer library. Cancer Res 2001, 61:2055-2061.

50. Giger OT, Lacoste E, Honegger C, Padberg B, Moch H, Varga Z: Expression of the breast differentiation antigen NY-BR-1 in a phyllodes tumor of the vulva. Virchows Arch 2007, 450:471-474.

51. Seil I, Frei C, Sultmann H, Knauer SK, Engels K, Jager E, Zatloukal K, Pfreundschuh M, Knuth A, Tseng-Chen Y, et al: The differentiation antigen NY-BR-1 is a potential target for antibody-based therapies in breast cancer. Int I Cancer 2007, 120:2635-2642.

52. Jager D, Karbach J, Pauligk C, Seil I, Frei C, Chen YT, Old L, Knuth A, Jager E: Humoral and cellular immune responses against the breast cancer antigen NY-BR-1: definition of two HLA-A2 restricted peptide epitopes. Cancer Immun 2005, 5:11.

53. Theurillat JP, Zurrer-Hardi U, Varga Z, Storz M, Probst-Hensch NM, Seifert B, Fehr MK, Fink D, Ferrone S, Pestalozzi B, et al: NY-BR-1 protein expression in breast carcinoma: a mammary gland differentiation antigen as target for cancer immunotherapy. Cancer Immunol Immunother 2007, 56:1723-1731.

doi:10.1186/1471-2407-13-271

Cite this article as: Balafoutas et al:: Cancer testis antigens and NY-BR-1 expression in primary breast cancer: prognostic and therapeutic implications. BMC Cancer 2013 13:271.

\section{Submit your next manuscript to BioMed Central and take full advantage of:}

- Convenient online submission

- Thorough peer review

- No space constraints or color figure charges

- Immediate publication on acceptance

- Inclusion in PubMed, CAS, Scopus and Google Scholar

- Research which is freely available for redistribution 